\title{
DEVELOPMENT OF IMAGE PROCESSING METHOD FOR HIGH QUALITY PIPELINE INSPECTION
}

\author{
Wondae Kim \\ Assistant Professor, Inha Technical College \\ kimwd@inhatc.ac.kr
}

\author{
Hwan-kook Hwang, Myung Jin Chae \\ Senior Researcher \\ Korea Institute of Construction Technology \\ \{hkhwang, Chae\}@kict.re.kr
}

\begin{abstract}
Sewer pipelines are buried underground and often forgotten until serious failure happens. Effective and reliable sewer system management is very important for the safe and clean environment. In order to maintain the intended capacity of pipes, effective maintenance based on accurate condition assessment is required. This research suggests a new method that uses digital still images to overcome the limitations of common CCTV pipeline inspection systems. As a result high quality images are obtained for accurate and effective condition assessment of pipelines.
\end{abstract}

\section{KEYWORDS}

Sewer Inspection, Digital Image Processing

\section{INTRODUCTION}

According to the Ministry of Construction and Transportation of Korea (MOCT), 819,558-km of roads and seven major underground infrastructures (water main, sewer lines, large area water main, gas, heat, electricity, and communication lines) should be electrically filed in a form of database. Out of 81,9558-km lines, $88.4 \%(724,311 \mathrm{~km})$ has already been stored in DB and used by public and private users. About 320 million US dollars have been invested on the development of the GIS systems for this system development [1]. However, the DB includes only the quantitative data such as location of pipes, installed depth, material type, installation date, etc.

The qualitative data such as the condition of pipes is not usually included in the DB. Since the condition of pipelines is often more important than location of pipelines, an effective and efficient method to collect the pipeline condition is critical in pipeline management $\mathrm{DB}$.

The most common method of pipeline inspection is using a remote-controlled-machine equipped with a CCTV (Closed Circuit TV), which, however, has many limitations to accurately inspect pipeline condition. In this research, a new approach to inspect the pipeline is developed. The suggested system takes pictures of inner surface of the pipe and produces high quality unwrapped images.

\subsection{Limitations of Typical CCTV Inspection}

In case of a typical CCTV, since the camera looks at the end point of the pipe, the locations of the defects and distance-readings are often different. In addition, the quality and accuracy of the inspection is highly dependent on the operator's skill and experience (Fig. 1). Various difference light conditions and the angle of the camera lens make various optical conditions making the inspection unreliable and unstable. The skill of the operator is the major factor of the accuracy and completeness of the inspection result.

Although recent pan-and-tilt cameras have overcome some limitations of simple front-viewing CCTV probes, still the operator's 

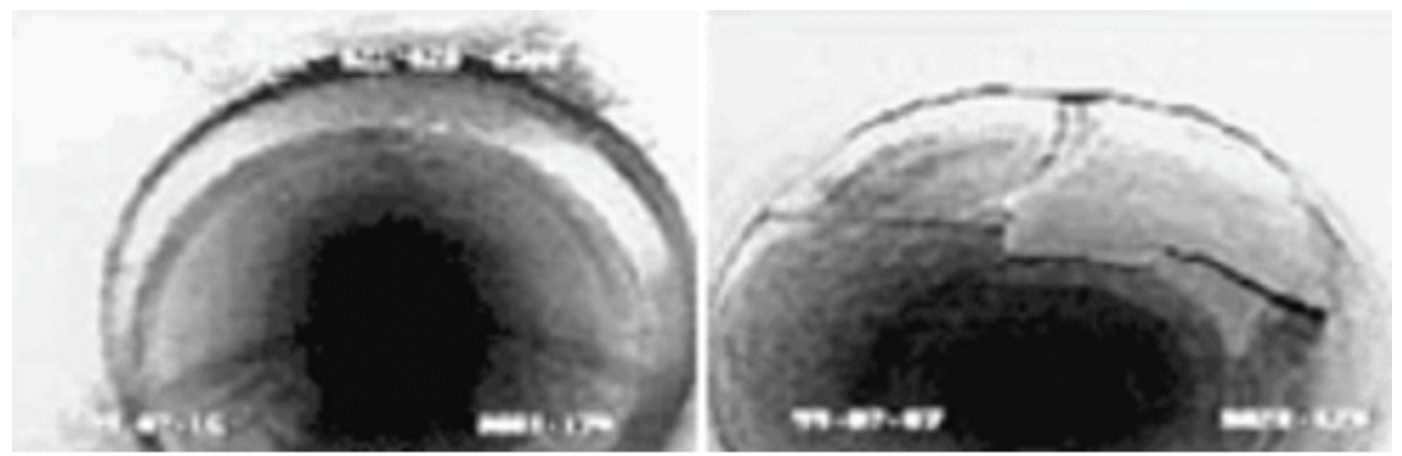

Figure 1 Stop and Pan-and-Tilt Images

skill is very important because the probe has to be stopped for pan-and-tilting.

Whether it is a front-view only camera or a pan-and-tilt camera, the quality and accuracy of the inspection highly depend on the skill and the experience of the operator. If the operator misses a defect, i.e., no stopping and pan-and-tilting, the defect would not be recorded and forgot until a new inspection is performed.

OYO-USA has developed a new type of sewer inspection system called SSET, which is equipped with a fish-eye lens to get a direct view to the pipe wall. It uses single line of image pixels to construct unwrapped circumferential images of inner surface of pipe wall. However, the fish-eye lens method for digital pipe line scanning had limitations in image quality and scanning speed.

\section{DEVELOPMENT OF DIGITAL SCANNER FOR SEWER PIPELINE INSPECTION}

\subsection{Image Acquisition and Processing}

In this research, a new approach is developed to overcome the limitations of SSET. The developed system uses larger areas of side view and constructs high quality stitched side view images.

As shown in the Figure 2, a plastic pipe was built to test the image acquisition system. Numbers are printed on the pipe wall to indicate the right location and stitching discrete images. Images are taken in several discrete blocks and unwrapped image result is presented in the Figure 3 and Figure 4.

\subsection{One Lens System for Both Front and Side View}

In order to obtain front view as well as side view simultaneously, and for better efficiency, one-lens system is developed, which is equipped with a common digital camera and specially designed mirror system to take both side and front view at the same time (Fig. 5).

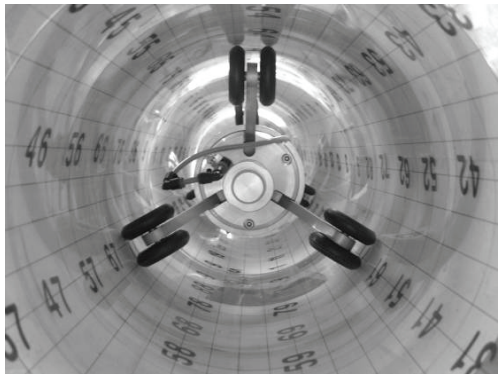

Figure 2 Image Acquisition Test Pipe

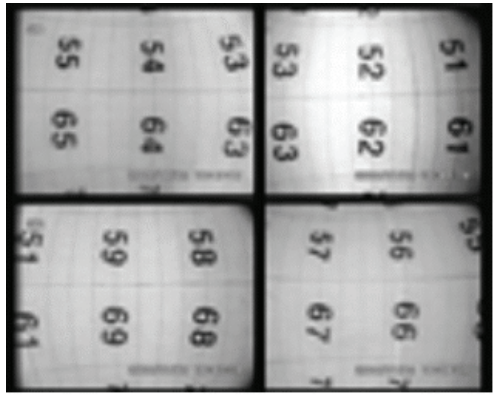

Figure 3 Before Image Stitching

Initially, before the one lens one CCD system was developed 4CCD system was developed. Scanned images of inner surface of pipe wall are processed 
and opened on a 2-D domain and stitched carefully. Figure 5 shows the 4 CCD camera system for 3-D image mapped on 2-D space. Gray areas the scanned areas by 4 CCD cameras and dark areas are the overlapped area the stitching reference.

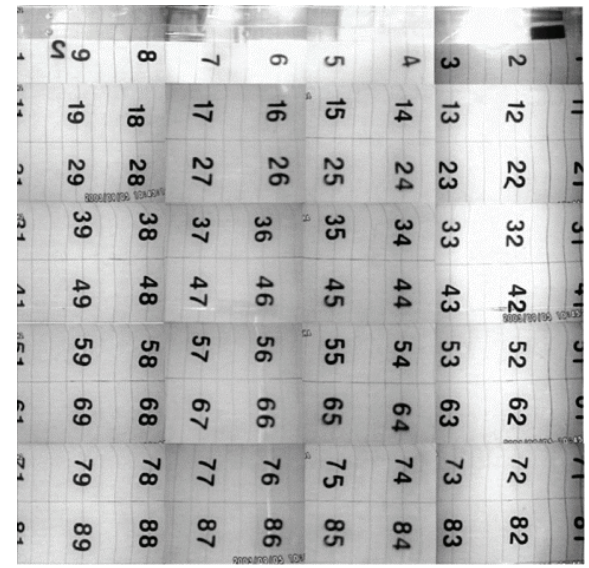

Figure 4 Stitched Image Mosaic

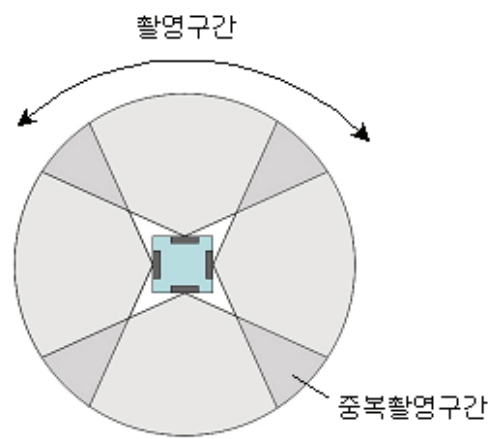

Figure 5 4CCD Scanning

However, this 4 CCD system has many difficulties in registration of the four different images. It required heavy load on manual image processing work.

One CCD system was developed based on the experience of 4CCD system. One CCD takes pictures both front and side view. In the Figure 6, CCD is located on the left side of the lens and the pipe object would be on the right. Side wall of inner surface of the pipe and the front view are obtained through this lens system. The resulting image is shown in Figure 9. Figure 7. shows the CCD camera and the developed lens.
When the camera scans the inner surface, the field of depth from the location of the camera to the object varies along with axial direction of the pipe. The distance calibration has to be performed to get the undistorted side view images. As in Figure 8, trapezoidal shaped images are obtained and it is process to be mapped on rectangular surface. $[2],[3]$

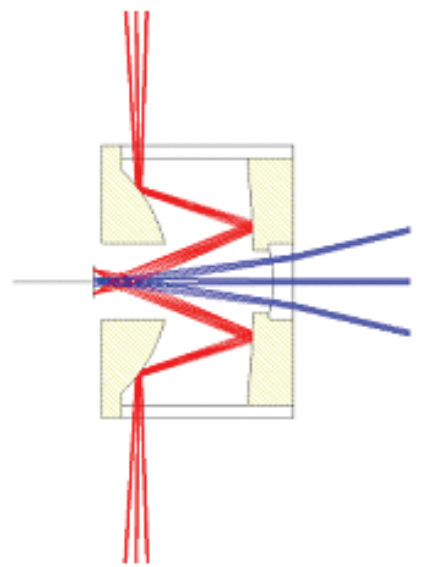

Figure 6 Design of the One-CCD System Lens

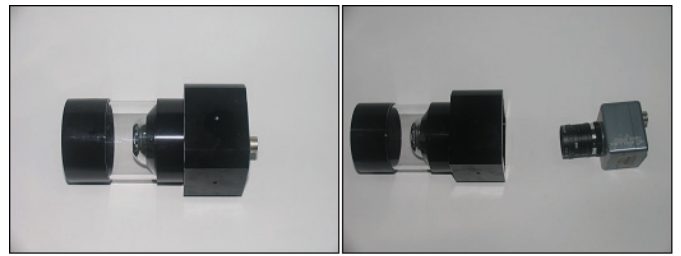

Figure 7 CCD Camera and Lens System

Once the images are calibrated it will be look like as shown in Figure 9. Straight lines in the pipe are shown straight. Camera collects certain distance of the pipe images. In order to develop a one continuous unwrapped image of the pipe, multiple stripes of the calibrated images are put together (registration) into one. The resulting image is shown in Figure 10. In this research, registration is completed by semi-automatically while distortion calibration is done automatically. In order for the perfect registration, a reference point (known objects) must be provided. However, the shapes in pipe wall usually do not have unique shape to be used as reference point. In the experiment, numbers on the pipe are good reference points, but 
in the real pipe, there is no number. Authors are current putting efforts to use distance reading as the registration reference. By use of laser distance meter, exact location of the probe is obtained and the image strips are stitched without reference point.
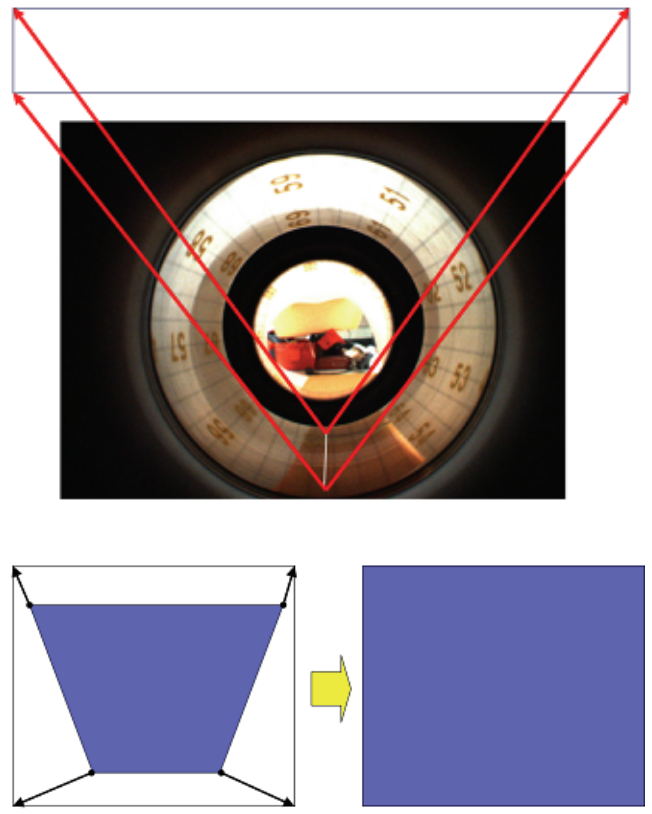

Figure 8 Image Distortion Calibration

\section{CONCLUSION}

In this research, a new system is developed by use of the image processing techniques. The image acquisition system is developed that inspects the inner surface of pipe. Side view unwrapping and stitching technology using image process techniques are developed which delivers high resolution image data.

Digital image scanning method is an innovative method for the pipe condition assessment. High quality images would bring dependable condition assessment of the pipes as well as the convenience of digital technology.

There were many barriers to make the unwrapped continuous image of the pipe line. Calibration of the distorted images is a difficult problem to solve, and the registration of processed strips of side wall is another difficult task. Since the pipe images usually does not have the distinct shapes to be used as reference point, instead of using reference point for the registration, accurate distance meter to get the exact location of the probe in the pipe is the better option. In this research project (will be continued until end of 2008) engineers will put effort mainly on the accuracy and reliability improvement of distance readings for the accurate image registration.

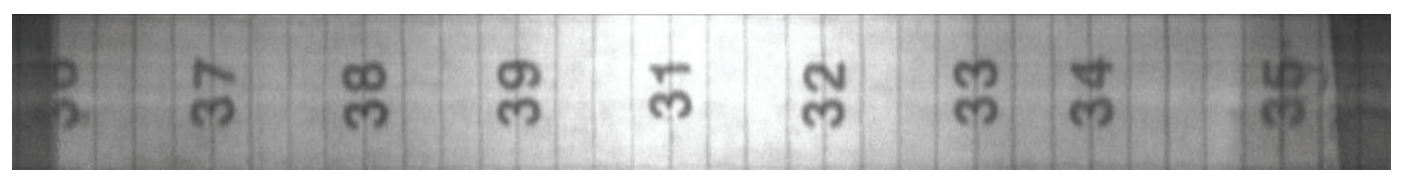

Figure 9 Adjusted Sample Image Strip

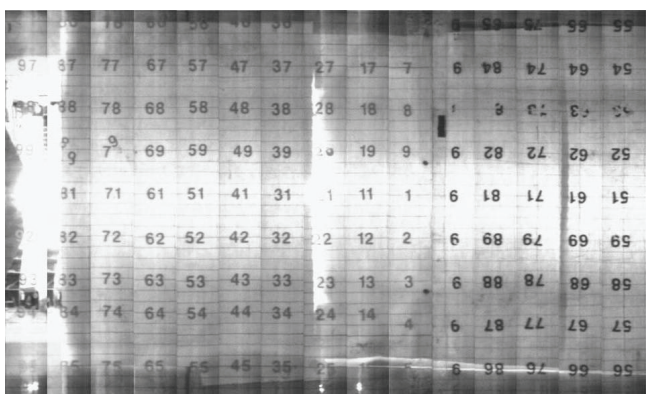

Figure 10 Side View Image Using One-Lens System

\section{ACKNOWLEDGMENT}

This research is supported by a grant (05 건설핵심 $\mathrm{C} 27$ ) of Ministry of Construction \& Transportation of Republic of Korea.

\section{REFERENCES}

[1] Lee, Shin, Park, Kim (2006) Water Supply and Sewerage, Goomi Books, Korea

[2] Kim, W., (2001) Photogrammetry, Inha Technical College Press, Korea

[3] Kim, W., (2003) Digital Photogrammetry, Dongin Press, Korea 